\title{
CoviD-19 What was the impact of COVID-19 on the foundation training programme in north Wales?
}

\author{
Authors: Sam Prince ${ }^{A}$ and Vedamurthy Adhiyaman ${ }^{B}$
}

The ongoing COVID-19 pandemic has affected postgraduate medical training across the UK. We surveyed foundation trainees in north Wales, receiving responses from 29 of 134. $80 \%$ felt that alterations to their training had negatively impacted their learning. $43 \%$ felt their progression to speciality training will be negatively affected. $70 \%$ did not think they were adequately involved in the decision making process.

KEYWORDS: foundation programme, postgraduate medical education, COVID-19

DOI: $10.7861 /$ fhj.2020-0026

\section{Introduction}

The UK foundation training programme is a two-year postgraduate training primarily undertaken by newly graduated medical students. Each year consists of three placements of 4 months. Due to the COVID-19 pandemic, the UK foundation programme either postponed or cancelled all the rotations that were due to happen in April 2020. ${ }^{1}$ As a result, the training programmes and the planned moves which were designed to help the trainees to choose their career were disrupted. We carried out a survey to assess the impact of this decision on foundation trainees, their feelings, and how they thought this might affect them in the future.

\section{Methodology}

We conducted a survey of foundation trainees across the three hospitals in north Wales, using an online survey system in June 2020. The survey was disseminated through their hospital email and social media.

Results

29 out of 134 trainees responded, of which 21 were foundation year 2 trainees. Only one trainee rotated in April as planned, and

Authors: Afoundation year 3 doctor, Glan Clwyd Hospital, Rhyl, UK; ${ }^{B}$ Consultant geriatrician, Glan Clwyd Hospital, Rhyl, UK two were redeployed to an area different from their current or intended placement. Two trainees were rotated once the perceived first local peak of COVID-19 had passed and 24 did not move remained in their second rotation for 8 months.

$80 \%$ of the trainees felt that the decisions taken due to the COVID-19 pandemic had negatively affected their learning. $43 \%$ felt that alteration to their training programme would negatively affect their progression to speciality training. Involvement of trainees in the decision making varied between the hospitals surveyed ranging from $55 \%, 20 \%$, and $0 \%$. Discussion with educational supervisors revealed that none of them were involved in these decisions.

\section{Discussion}

The decision to cancel or postpone the rotation had a significant negative impact on the foundation trainees' perception and experience towards training. Many were unhappy that they had missed out on adequate clinical exposure in different specialities. Because the foundation programme ended in August 2020, there will be no time to restructure future placements and the missed placements would always remain missed. Some specialities (for example general practice) do not offer stand-alone short-term employment at the foundation trainee level. Therefore, it would be impossible for trainees who had missed the opportunity to rotate to gain any experience in those specialities at a later date.

Some trainees were aggrieved by perceived unfairness between colleagues, with some rotating as planned while others could not because they were either redeployed to COVID-19 wards or asked to remain in the same placement for 8 months. In total, $70 \%$ of the trainees felt there was lack of involvement regarding alterations to their rotations. This is a major concern because it gives the impression that they were undervalued and their opinions did not count.

Many trainees shared the view that rotation would have been possible, and that their services were never so busy so as to make that unsafe.

A similar survey was undertaken in the East of England and East Midlands. Although not directly associated, similar enough questions were asked to allow comparisons to be drawn. Rotation into third placement was similar at $90 \%$ in north Wales compared to $89 \%$ in east England. A marked difference was noted in 
re-deployment rates, with just $7 \%$ of north Wales trainees redeployed compared to $59 \%$ in east England. ${ }^{2}$ This could partially be explained by differing demands geographically. Similar themes were identified in both areas. Missing out on specialty experience is a common complaint, as are the adverse effects on future opportunities and progression to further training.

\section{Conclusion}

In future, there should be more involvement of the trainees and their educational supervisors in the decision-making processes relating to changes to training, with the aim of deciding what would be best for the trainees and the organisation rather than making a blanket policy. Training will always be an acceptable casualty of pandemics, but it could be argued that the demand on the hospitals in north Wales had not been severe enough to justify this. The GMC survey that is currently underway should provide more information on the impact of COVID-19 on trainees and trainers.

\section{References}

1 UK Foundation Programme. Impact of COVID-19 on the UK Foundation Programme 2020. UKFPO, 2020. https://foundationpro gramme.nhs.uk/covid-19/ [Accessed 23 July 2020].

2 Sasitharan A. COVID-19: The impacts on foundation training in district general hospitals in Eastern England and the East Midlands. Clin Med 2020, in press (doi: 10.7861/clinmed.2020-0592).

Address for correspondence: Dr S Prince, Ysbyty Glan Clwyd, Rhuddlan Road Bodelwyddan Rhyl LL18 5 UJ.

Email: sam.prince94@gmail.com 